\title{
Hallux Rigidus: Diagnosis and Management
}

\author{
Nurettin Heybeli ${ }^{1}$, Burak Günaydın ${ }^{2}$
}

\begin{abstract}
Hallux rigidus is the most common arthritis of the foot, characterized by dorsiflexion restriction with osteophyte formation. Patients complain of pain and loss of motion. Nonsurgical treatments can be tried successfully in early-stage patients. The surgical treatment options can be a joint-preserving technique or not. Arthrodesis has been the main treatment option for advanced-stage patients. Arthroplasty has also been an available option. There are other treatment options available for the treatment of hallux rigidus, and these are to be discussed in the present article, accompanied by the literature.

Keywords: Arthrodesis, Cheilectomy, Hallux limitus, Hallux rigidus, Hemiarthroplasty, Interpositional arthroplasty, Resection arthroplasty, Synthetic cartilage implants, Total arthroplasty.

Journal of Foot and Ankle Surgery (Asia Pacific) (2020): 10.5005/jp-journals-10040-1125
\end{abstract}

\section{INTRODUCTION}

Hallux rigidus is a degenerative disease characterized by the dorsiflexion restriction of the first metatarsophalangeal (MTP) joint. Osteoarthritis is most commonly seen at the first MTP joint among foot joints. It is a relatively common problem and is seen in $25 \%$ of patients over 50 years of age in different stages in the public.

\section{Etiology and InCidence}

The primary etiology of the disease is unknown. Trauma creates a predisposition, and a direct trauma or more commonly repetitive microtrauma may cause the disease. It may accompany deformities, such as, flat, elevated, or chevron-shaped first MTP joint, metatarsus primus elevatus, hallux valgus interphalangeus, and metatarsus adductus. It can also be seen as secondary to metabolic and rheumatic diseases.

It is more common in women than in men. ${ }^{2}$ Patients with hallux rigidus are seen bilaterally in $80 \%$, if they are followed for a sufficient time. Additionally, $80 \%$ of patients with bilateral hallux rigidus have a positive family history. ${ }^{3}$ Increases in the degenerative arthritic changes were reported with advancing age. ${ }^{4}$

\section{Symptoms and Findings}

Hallux rigidus is a disease initially characterized by pain, swelling, synovitis of the MTP joint, and restricted dorsiflexion. The dorsiflexion restriction develops due to the contractures in the plantar part of the MTP joint capsule and dorsal osteophyte at the metatarsal head. The limitation of movement is seen as a problem during the liftoff phase of the walk, as well as running, walking uphill, and wearing high heels. Pain at the dorsum of the distal first metatarsal due to the dorsal osteophyte is a prominent problem stated by the patients while wearing shoes. Swelling and stiffness are other common complaints. Generally, there is an enlargement of the joint due to osteophytes and soft tissue swelling. Hyperesthesia, tingling, or positive Tinel's signs are noteworthy findings caused by the pressure on the dorsomedial cutaneous branch of the superficial peroneal nerve with the dorsal/dorsolateral osteophytes. When axial compression to the MTP joint is applied within the middle of

\footnotetext{
1,2Department of Orthopaedics and Traumatology, School of Medicine, Namık Kemal University, Tekirdağ, Turkey

Corresponding Author: Nurettin Heybeli, Department of Orthopaedics and Traumatology, School of Medicine, Namık Kemal University, Tekirdağ, Turkey, Phone: +90-532-286-1446, e-mail: nurettin.heybeli@ gmail.com

How to cite this article: Heybeli N, Günaydın B. Hallux Rigidus: Diagnosis and Management. J Foot Ankle Surg (Asia Pacific) 2020;7(2):64-69.

Source of support: Nil

Conflict of interest: None
}

the range of motion, positive compression (grind) test is mentioned as a result of pain. The positive grind test shows that arthritis in the MTP joint is at an advanced level, and the positive finding of this examination in patients is relatively a contraindication for cheilectomy. During physical examination, the metatarsosesamoid joint must also be evaluated. ${ }^{1-4}$

\section{Radiologic Evaluation}

Weight-bearing anteroposterior (AP), lateral, and oblique radiographs are usually sufficient for the diagnosis of this disease. AP radiographs are especially useful for evaluating the medial and lateral osteophytes and narrowing of the joint space. The lateral radiograph shows the size of the dorsal osteophytes. In advanced cases, dripping candle wax appearance can be detected on the lateral radiography. Oblique $\mathrm{X}$-ray is used to evaluate the narrowed joint space. In advanced disease stages, subchondral cysts, sclerosis of the first metatarsal head, and enlargement in the proximal phalanx base are appointed. Magnetic resonance imaging (MRI) and computerized tomography (CT) are generally not required. In selective cases, MRI may be helpful to assess osteochondral injuries. ${ }^{5}$

Hallux rigidus disease was radiologically divided into three phases by Hattrup and Johnson in $1988 .{ }^{6}$ In 2003 , Coughlin and Shurnas developed a classification system, including clinical signs as well as radiological findings, and divided this disease into four stages. ${ }^{7}$

() The Author(s). 2020 Open Access This article is distributed under the terms of the Creative Commons Attribution 4.0 International License (https://creativecommons. org/licenses/by-nc/4.0/), which permits unrestricted use, distribution, and non-commercial reproduction in any medium, provided you give appropriate credit to the original author(s) and the source, provide a link to the Creative Commons license, and indicate if changes were made. The Creative Commons Public Domain Dedication waiver (http://creativecommons.org/publicdomain/zero/1.0/) applies to the data made available in this article, unless otherwise stated. 


\section{Treatment}

\section{Nonsurgical Treatment}

Conservative treatment can be applied depending on the patient's symptoms and the stage of osteoarthritis. Morton's type extension or carbon fiber plate orthoses can be used. These orthoses are effective by restricting finger movement. ${ }^{8}$ Treatment with rest and anti-inflammatory drugs can be prescribed. High-heeled rocker sole shoes can also be useful in some patients to improve walking. ${ }^{8}$ Shoes with large toe box can reduce the patients' symptoms by increasing the space for dorsal osteophytes, while reducing the pressure on the joint.

Steroid and sodium hyaluronic acid injections into the MTP joint can be done. However, the steroid injection provides a shortterm relief, but repeated injections may speed up the degenerative process. Physical therapy is recommended for patients with shorter hamstring and/or Achilles tendons.

In a review by Grady et al., evaluating 772 hallux rigidus cases, patients undergoing surgery and conservative treatment were evaluated. It was found that $55 \%$ of the patients were successfully treated with conservative treatment. They also found that $13 \%$ of the patients who failed conservative treatment refused surgical intervention or were not suitable candidates for surgical intervention. ${ }^{9}$ In a study conducted by Smith et al., 22 patients (24 feet) followed for minimum 12 years, $75 \%$ of the cases would "still chose not to have surgery" if they had to make the decision again despite significant deterioration in joint space noted radiographically. ${ }^{10}$

\section{Surgical Treatment}

There are many surgical treatment options. It can be divided into two as those who do preserve and do not preserve the joint.

\section{Joint-preserving Surgical Procedures}

Joint debridement and synovectomy, dorsal cheilectomy, proximal phalanx dorsal osteophyte excision, and dorsal closed wedge proximal phalanx osteotomy or wide plantar release and microfracture methods combined with cheilectomy can be considered.

Joint debridement and synovectomy is a technique used in early-stage patients. Dorsal cheilectomy is the most commonly used technique in mild and moderate hallux rigidus patients. This technique includes dorsal osteophyte excision and partial excision of the dorsal face of the metatarsal head. ${ }^{11}$ An important trick in this technique is to remove less than one-third of the dorsal metatarsal head, to prevent the dorsal dislocation of the proximal phalanx. ${ }^{12}$ Generally, this technique reduces the dorsal compression of the proximal phalanx. In a retrospective study conducted by Coughlin and Shurnas in 2004, they found $92 \%$ improvement in AOFAS scoring, as well as decreased pain and increased function in patients with a follow-up of average 9.6 years after cheilectomy. ${ }^{13}$

In a retrospective study conducted by Easley et al., in 75 feet of 57 patients with a minimum follow-up of 3 years, who were treated with medial-approach dorsal cheilectomy for hallux rigidus, the dorsal osteophyte recurrence was seen in 21 feet, 9 of which were

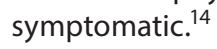

With the advances in endoscopic techniques in recent years, arthroscopic- and fluoroscopic-assisted percutaneous cheilectomies have also been reported. In a study with an average of 3 years of follow-up conducted by Steven et al., the authors reviewed open cheilectomy of 38 patients and minimally invasive cheilectomy of 133 patients. The complication rate in the open group was $2.6 \%$ (1 patient), while it was $11.3 \%$ (15 patients) in the minimally invasive group. In the follow-up of patients, the necessity of fusion surgery was $2.6 \%$ (1 patient) in patients who underwent open cheilectomy and $12.8 \%$ (17 patients) in those who underwent the minimally invasive technique. Although better cosmetic and early recoveries are achieved in the minimally invasive group, the need for fusion surgery was found to be high, which is an important challenge that needs to be addressed..$^{15}$

Moberg closed wedge (proximal phalanx) osteotomy and cheilectomy: Dorsal closed wedge osteotomy increases the dorsal MTP joint space. ${ }^{16}$ Osteotomy pushes the dorsal face of the phalanx beyond the dorsal face of the first metatarsal head. It reduces the compression force in the dorsum of the metatarsophalangeal joint. This technique is indicated in young and active patients, in patients with moderate to severe hallux rigidity, and in patients with limited dorsiflexion in the first metatarsophalangeal joint. ${ }^{17}$ If the patient has normal dorsiflexion in the first metatarsophalangeal joint, this surgical treatment option is contraindicated. Similarly, in the elderly, low-activity patients and if there is no plantar flexion in the first MTP joint, it is contraindicated. In patients with hallux rigidus, when Moberg osteotomy is combined with cheilectomy, successful results were often described, especially in patients where conservative treatment failed..$^{18}$ In a 22-year follow-up study of Citron and Neil, 10 feet of 8 patients underwent dorsal wedge osteotomy of the proximal phalanx and cheilectomy. In the follow-up, symptoms were resolved in five feet, while pain was present in others, walking was not restricted, and one foot required MTP fusion. The authors concluded that dorsal wedge osteotomy is useful in a case series with a long-term follow-up. ${ }^{19}$ In a prospective study conducted by Kilmartin, $89 \%$ of patients were reported as satisfied with this technique. It had been necessary to remove the implants applied in $8 \%$, and the AOFAS metatarsophalangeal-interphalangeal joint scale showed an average improvement of 45 points. ${ }^{20}$ O'Malley et al. performed proximal phalanx dorsal wedge osteotomy in 81 hallux rigidus cases. They found $85 \%$ satisfaction in 4-year followups. Arthrodesis surgery was required in 4 patients; implants had to be removed in 3 patients. ${ }^{17}$

Dorsal cheilectomy, wide plantar release, and microfracture: It is indicated selectively in stage 2 and stage 3 patients without joint stiffness and is contraindicated in stage 3 and stage 4 patients where joint stiffness is present.

Proximal phalanx dorsal osteophyte excision: This technique is applied with dorsal cheilectomy if osteophyte formation is present in the proximal phalanx.

\section{Joint-sacrificing Techniques}

Surgeries that do not protect the joint include arthrodesis, joint resection or interpositional arthroplasties, and hemi- or total prosthetic arthroplasties. Although arthrodesis is considered as the gold standard, movement limitation develops. The use of arthroplasty, on the contrary, protects movement in the joint after surgery. Arthroplasty options can be summarized as total metatarsophalangeal arthroplasty, ${ }_{1}^{21,22}$ hemiarthroplasty, ${ }^{23}$ and joint resection and/or interpositional arthroplasty. ${ }^{24,25}$

MTP joint resection arthroplasty/interpositional arthroplasty: Interpositional arthroplasty is applied together with Keller resection arthroplasty. It involves placing biological spacer in the area after Keller-type resections. In interpositional arthroplasty, the dorsal capsule and extensor hallucis brevis are released most frequently 
and placed in the joint space to fill the space. ${ }^{26}$ Metatarsalgia and weakness can be seen as complications. By modifying the technique, less proximal phalanx can be resected and flexor hallucis brevis can be preserved. Less instability can be seen with this modified technique. In a study conducted by Akgün et al., authors reported that they could not detect cock-up toe deformity and push-off weakness by applying this modified technique. ${ }^{27}$ It is especially preferred for patients with low activity levels. In a metaanalysis performed with the inclusion of 15 publications in 2018, clinical results and complication rates of interpositional arthroplasty were evaluated in patients with intermediate and advanced hallux rigidus. ${ }^{28}$ The authors concluded that interpositional arthroplasty can be performed with acceptable clinical results in patients with intermediate and advanced hallux rigidus. ${ }^{28}$ In the study of Coughlin and Shurnas with 7 feet of 7 patients, they used the gracilis tendon as biological tissue and reported decreased pain and function at an average follow-up of 42 months. ${ }^{29}$

\section{Arthroplasty Techniques}

Hemiarthroplasty is performed as proximal phalanx basis hemiarthroplasty or distal metatarsal hemiarthroplasty. In the late years, it is more commonly applied to the distal metatarsal. It is preferred in patients with advanced osteoarthrosis and who do not accept arthrodesis. In the early days, silastic implants were common, then metal implants became popular, and finally nowadays synthetic cartilage implants are used. High rates of osteolysis and immune reactions were observed in silastic implants. There were large bone losses in failures with these implants; therefore, it was a challenge to accomplish a solid arthrodesis. ${ }^{30}$

Total implants usually showed encouraging early-term results. For example, in a study with an average follow-up of mean 47 months, the authors concluded on their total joint system as "appeared to be satisfactory," although a 5.5\% revision and 33\% implant depression were detected. ${ }^{31}$ In a randomized controlled trial, Gibson and Thompson found out that outcomes after arthrodesis were better than those after arthroplasty. "Unacceptably high incidence of loosening of the phalangeal components" was noted, and they concluded that arthrodesis was "clearly" preferred by most patients. ${ }^{32}$ However, reviewing the literature, we still see a few reports of "good functional results and good satisfaction levels" in patients who underwent total arthroplasty in hallux rigidus patients, but these are almost always with short-term follow-ups. ${ }^{33}$ In a recent metaanalysis performed in 2019, implant arthroplasty and arthrodesis were compared. The conclusion was they both led to similar clinical outcomes, patient satisfaction, reoperation rates, and complication rates, whereas pain was significantly lower in arthrodesis patients. ${ }^{34}$ Hemiarthroplasty and total joint arthroplasty in hallux rigidus were also compared in another meta-analysis in $2019 .{ }^{35}$ In this metaanalysis, hemi-surface implants were found to have better results in terms of AOFAS and EHA compared to total joint arthroplasty. ${ }^{35}$

\section{Synthetic Cartilage Implants}

A recently marketed synthetic cartilage implant showed conflicting results in two different studies. In a study by Glazebrook et al., in $14 / 152(9.2 \%)$ patients, implants had to be removed in the first 2 years of follow-up, and arthrodesis was applied. In the 2-to-5-year period, in 9/119 (7.6\%) patients, implant removal and arthrodesis surgery were required. The authors concluded that the implant continues to be an appropriate treatment option to reduce pain, improve function, and maintain movement. ${ }^{36}$ In another study involving 64 hallux rigidus patients, Spencer et al. found out that
$20 \%$ of these patients required surgery again and, in $8 \%$, arthrodesis was applied. ${ }^{37}$ Even in the early follow-up, moderate pain, loss of physical activity, and patient satisfaction were reported. The authors gave a word of caution stating that while applying this technique, attention should be paid to patient selection and patients should be informed about postoperative limitations. ${ }^{37}$ The good thing with the reoperation might be, since this implant is applied by removing $8-10 \mathrm{~mm}$ of bone distal to the metatarsal, less difficulty is encountered when arthrodesis is needed.

\section{Arthrodesis}

This is the gold standard in patients with advanced hallux rigidus. Although the first MTP joint arthrodesis is the most effective method of relieving pain, movement limitation may not be preferred by some patients. It is the treatment option that should be preferred especially if the patient has hallux valgus, hallux varus, as well as rheumatoid rigidus, rheumatic diseases, and neuromuscular disorders. While preparing joint surfaces in this technique, it can be prepared flat or conical, which may need additional instruments. For fixation, plates or screws or both can be used. Although some authors recommend fewer degrees when applying arthrodesis, $10-15^{\circ}$ of dorsiflexion of the hallux is recommended with $10-15^{\circ}$ of valgus and neutral rotation. ${ }^{38,39}$ If there is advanced osteoarthrosis in the first interphalangeal and tarsometatarsal joint, arthrodesis is contraindicated.

In the fusion of the first MTP joint, the most stable technique was found to be provided by oblique lag screws and a plate applied from the dorsal. ${ }^{40}$ Goucher and Coughlin in a prospective study of 54 feet of 50 hallux rigidus patients used a lag screw and dorsal plate with conical reamers to obtain arthrodesis. Short-term follow-ups showed $96 \%$ satisfaction and $92 \%$ union rates. The authors found arthrodesis as "reliable and reproducible" using this technique. ${ }^{41}$

\section{SUMmary}

Hallux rigidus is the most common arthritis of the foot that causes progressive degeneration, the etiology of which cannot be fully explained in every patient. Patients often complain of pain, swelling, and movement limitations. Loss of joint motion and pain are the hallmarks. The motion problem is in the sagittal plane, especially with dorsiflexion restriction. If the disease is in a very advanced stage, it can cause movement restriction in the entire metatarsophalangeal joint. In the early phases of patient care, nonsurgical treatments can be used. In many patients, conservative treatment can be successfully applied at an early stage. Cheilectomy is performed with successful results in stage 1 and stage 2 hallux rigidus. In advanced-stage patients, surgical options are divided into two categories: the ones that protect the joint and the ones that do not. Arthrodesis is the gold standard treatment in advanced-stage patients. While arthrodesis is the most effective method of relieving pain, it causes limitation of movement. Most patients are eager for arthroplasty, to protect and improve the joint range of motion. Therefore, we can observe many arthroplasty options in the literature. However, further, larger case series are needed to demonstrate the superiority of these surgical techniques over arthrodesis.

\section{Case Description}

A 54-year-old woman presented with continuing pain and instability of her great toes after a bilateral first MTP joint prosthesis total operation done elsewhere. 
On inspection, hallux valgus deformity and swelling of the joint were observed, and on physical examination, the range of motion was limited, the patient complained of pain, and the instability was also evident. The findings were more prominent on the right side.

On radiographic examinations, bilateral subluxations of the joint that was more evident on the right side were detected (Fig. 1).

A revision to arthrodesis was planned. After the exposure, the implants were found to be misaligned and subluxated. There was no osteointegration, so the implants could easily be removed. The fibrous tissue around and at the nonunion site between the bone and implants was thoroughly debrided to expose spongious bone for healing (Fig. 2)

Upon removal of the implants, the size of the defect necessitated a tricortical autogenous iliac bone graft. Shortening was prevented using the graft for intercalary reconstruction. The graft was temporarily fixed with an intramedullary K-wire, and a dorsal lowprofile titanium plate was applied (Fig. 3).
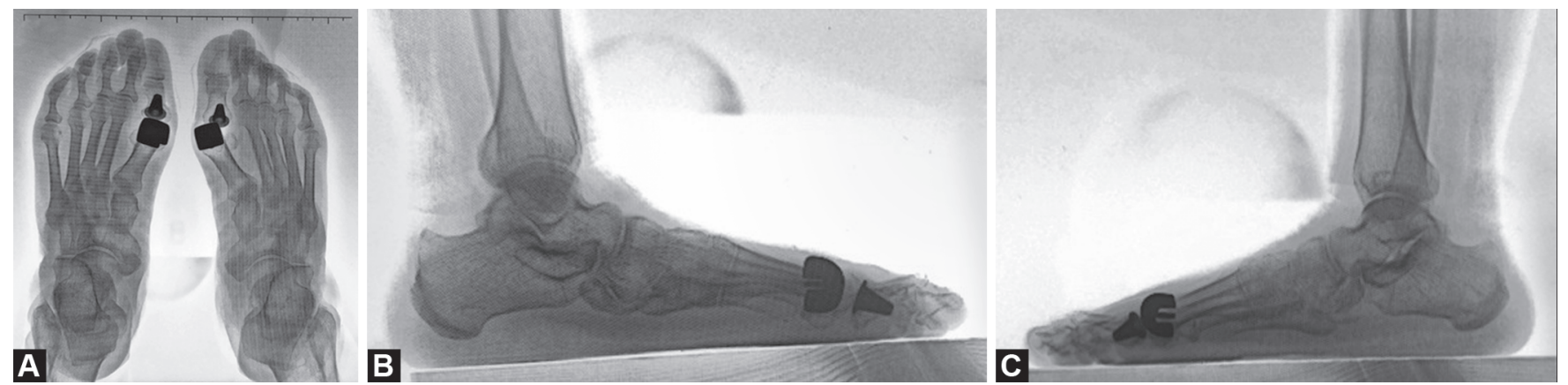

Figs $1 \mathrm{~A}$ to $\mathrm{C}$ : (A) Anteroposterior radiograph of the patient. Note the subluxation; (B) Lateral radiograph of the left side; (C) Lateral radiograph of the right side
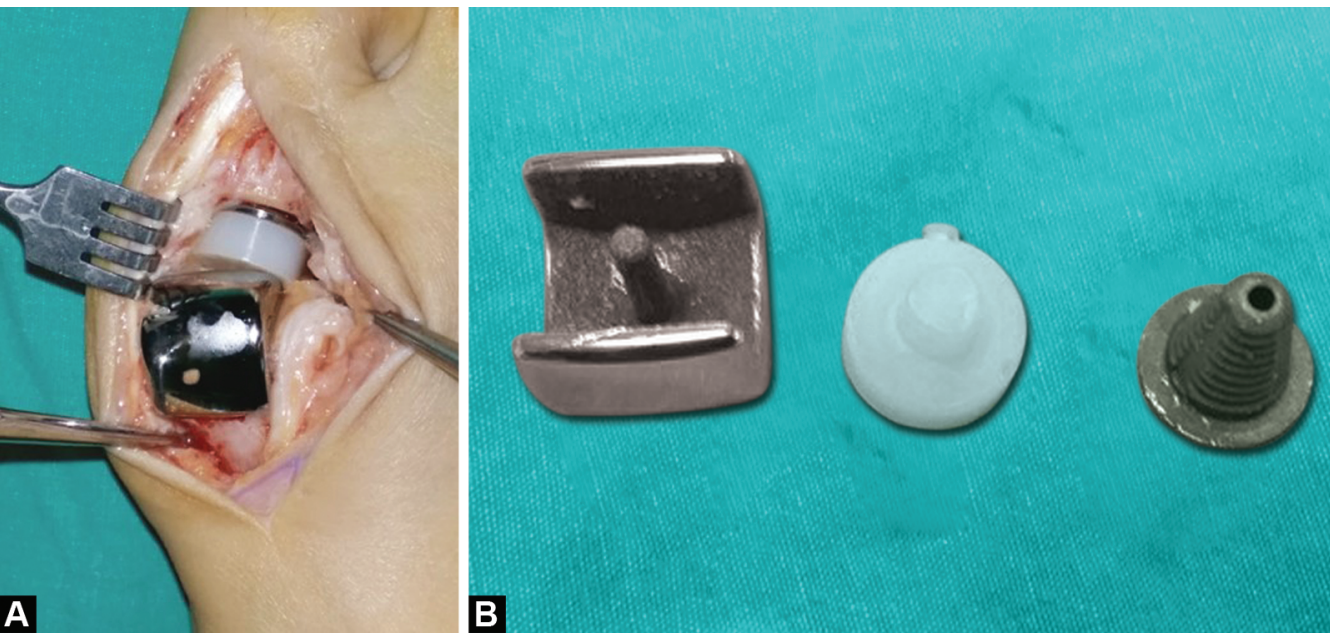

Figs 2A to C: (A) Exposure of the metatarsophalangeal joint showing subluxated, unstable prosthetic components; (B) Back sites of the implants showed no signs of osteointegration; (C) Meticulous and thorough debridement of the fibrotic tissues to obtain healing
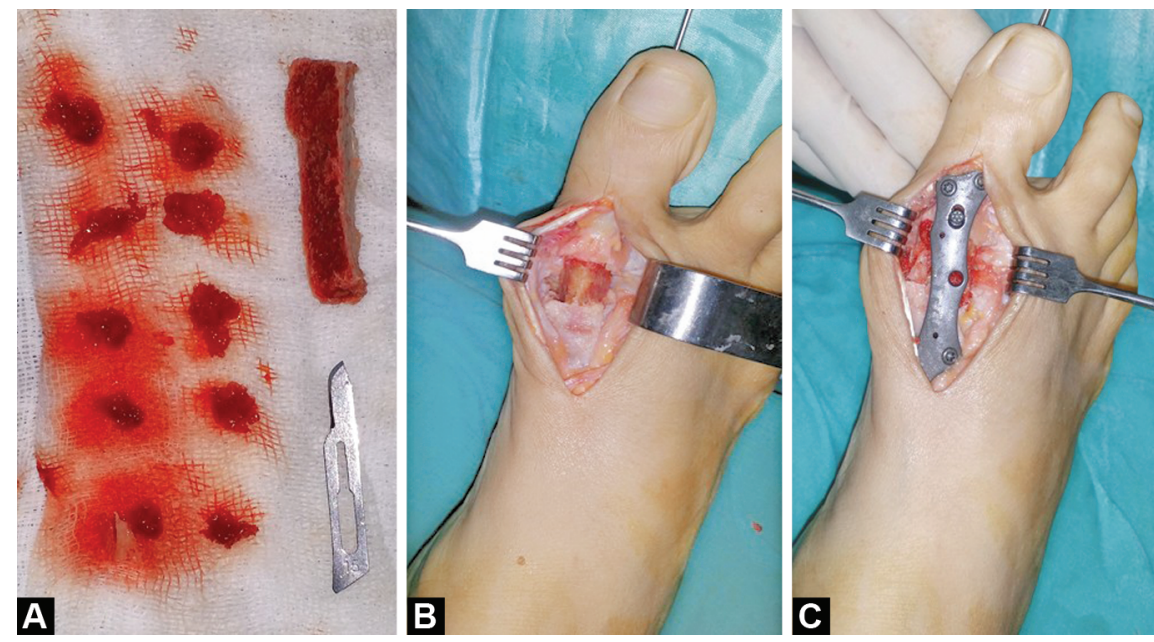

Figs 3A to C: (A) Tricortical autogenous iliac bone graft with additional bone chips; (B) Graft in place, temporarily fixed with an intramedullary K-wire; (C) Final fixation with a dorsal low-profile titanium plate and screws 

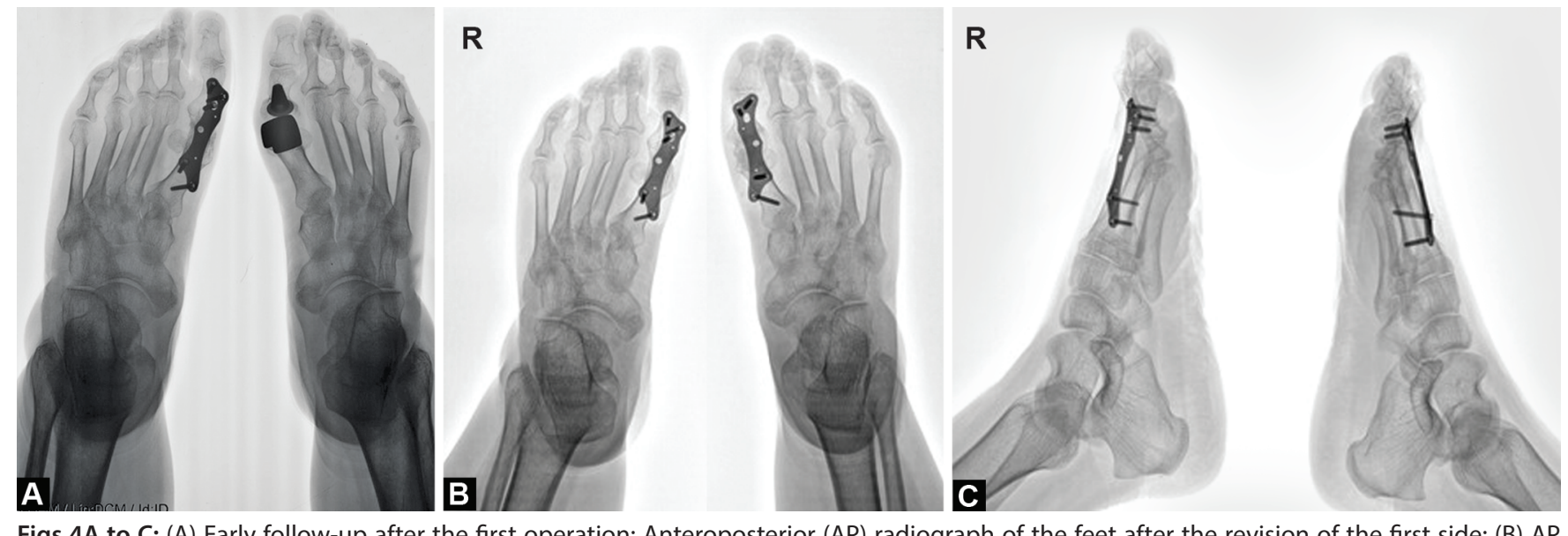

Figs 4A to C: (A) Early follow-up after the first operation: Anteroposterior (AP) radiograph of the feet after the revision of the first side; (B) AP radiograph of the feet after the revision of the other side; $(C)$ Lateral radiograph of the feet after the revision of the other side

A solid union was achieved on the right side in a few months. The patient being satisfied with the revision to arthrodesis on her right side demanded the same revision technique in favor of arthrodesis to her left side as well (Fig. 4).

\section{References}

1. Ho B, Baumhauer J. Hallux rigidus. EFORT Open Rev 2017;2(1):13-20. DOI: 10.1302/2058-5241.2.160031.

2. Mann RA, Thompson FM. Arthrodesis of the first metatarsophalangeal joint for hallux valgus in rheumatoid arthritis. J Bone Joint Surg Am 1984;66(5):687-692. DOI: 10.2106/00004623-19846605000006.

3. Coughlin MJ, Shurnas PS. Hallux rigidus: demographics, etiology, and radiographic assessment. Foot Ankle Int 2003;24(10):731-743. DOI: $10.1177 / 107110070302401002$.

4. Mann RA, Clanton TO. Hallux rigidus: treatment by cheilectomy. J Bone Joint Surg Am 1988;70(3):400-406. DOI: 10.2106/00004623198870030-00012.

5. Schweitzer ME, Maheshwari S, Shabshin N. Hallux valgus and hallux rigidus: MRI findings. Clin Imaging 1999;23(6):397-402. DOI: 10.1016/ s0899-7071(00)00167-4.

6. Hattrup SJ, Johnson KA. Subjective results of hallux rigidus following treatment with cheilectomy. Clin Orthop Relat Res 1988;226(226): 182-191. DOI: 10.1097/00003086-198801000-00025.

7. Coughlin MJ, Shurnas PS. Hallux rigidus: grading and long-term results of operative treatment. J Bone Joint Surg Am 2003;85(11): 2072-2088. DOI: 10.2106/00004623-200311000-00003.

8. Kunnasegaran R, Thevendran G. Hallux rigidus nonoperative treatment and orthotics. Foot Ankle Clin 2015;20(3):401-412. DOI: 10.1016/j.fcl.2015.04.003.

9. Grady JF, Axe TM, Zager EJ, et al. A retrospective analysis of 772 patients with hallux limitus. J Am Podiatr Med Assoc 2002;92(2): 102-108. DOI: 10.7547/87507315-92-2-102.

10. Smith RW, Katchis SD, Ayson LC. Outcomes in hallux rigidus patients treated nonoperatively: a long-term follow up study. Foot Ankle Int 2000;21(11):906-913. DOI: 10.1177/107110070002101103.

11. Mann RA, Coughlin MJ, DuVries HL. Hallux rigidus: a review of the literature and a method of treatment. Clin Orthop Relat Res 1979;142(142):57-63. DOI: 10.1097/00003086-19790700000010.

12. Heller WA, Brage ME. The effects of cheilectomy on dorsiflexion of the first metatarsophalangeal joint. Foot Ankle Int 1997;18(12):803-808. DOI: $10.1177 / 107110079701801209$.

13. Coughlin MJ, Shurnas PS. Hallux rigidus: surgical techniques (cheilectomy and arthrodesis). J Bone Joint Surg Am 2004;86(Pt 2):119-130. DOI: 10.2106/00004623-200409001-00003.
14. Easley ME, Davis WH, Anderson RB. Intermediate to long-term follow-up of medial-approach dorsal cheilectomy for hallux rigidus. Foot Ankle Int 1999;20(3):147-152. DOI: 10.1177/107110079902000302.

15. Stevens R, Bursnall M, Chadwick C, et al. Comparison of complication and reoperation rates for minimally invasive versus open cheilectomy of the first metatarsophalangeal joint. Foot Ankle Int 2020;41(1):31-36. DOI: $10.1177 / 1071100719873846$.

16. Hamid KS, Parekh SG. Clinical presentation and management of hallux rigidus. Foot Ankle Clin 2015;20(3):391-399. DOI: 10.1016/j. fcl.2015.04.002.

17. O'Malley MJ, Basran HS, Gu Y, et al. Treatment of advanced stages of hallux rigidus with cheilectomy and phalangeal osteotomy. J Bone Joint Surg Am 2013;95(7):606-610. DOI: 10.2106/JBJS.K.00904.

18. Roukis TS. Outcomes after cheilectomy with phalangeal dorsiflexory osteotomy for hallux rigidus: a systematic review. J Foot Ankle Surg 2010;49(5):479-487. DOI: 10.1053/j.jfas.2010.05.006.

19. Citron N, Neil M. Dorsal wedge osteotomy of the proximal phalanx for hallux rigidus. Long-term results. J Bone Joint Surg Br 1987;69(5): 835-837. DOI: 10.1302/0301-620X.69B5.3680354.

20. Kilmartin TE. Phalangeal osteotomy versus first metatarsal decompression osteotomy for the surgical treatment of hallux rigidus: a prospective study of age matched and conditionmatched patients. J Foot Ankle Surg 2005;44(1):2-12. DOI: 10.1053/j. jfas.2004.11.013.

21. Kim PJ, Hatch D, Didomenico LA, et al. A multicenter retrospective review of outcomes for arthrodesis, hemi-metallic joint implant, and resectional arthroplasty in the surgical treatment of end-stage hallux rigidus. J Foot Ankle Surg 2012;51(1):50-56. DOI: 10.1053/j. jfas.2011.08.009.

22. Lawrence BR, Thuen E. A retrospective review of the primus first MTP joint double-stemmed silicone implant. Foot Ankle Spec 2013;6(2):94-100. DOI: 10.1177/1938640012470715.

23. Erdil M, Bilsel K, Imren $Y$, et al. Metatarsal head resurfacing hemiarthroplasty in the treatment of advanced stage hallux rigidus: outcomes in the short-term. Acta Orthop Traumatol Turc 2012;46(4):281-285. DOI: 10.3944/aott.2012.2795.

24. Schenk S, Meizer R, Kramer R, et al. Resection arthroplasty with and without capsular interposition for treatment of severe hallux rigidus. Int Orthop 2009;33(1):145-150. DOI: 10.1007/s00264-007-0457-z.

25. Love TR, Whynot AS, Farine I, et al. Keller arthroplasty: a prospective review. Foot Ankle 1987;8(1):46-54. DOI: 10.1177/107110078700800110.

26. Mackey RB, Thomson AB, Kwon O, et al. The modified oblique Keller capsular interpositional arthroplasty for hallux rigidus. J Bone Joint Surg Am 2010;92-A(10):1938-1946. DOI: 10.2106/JBJS.I.00412.

27. Can Akgun R, Sahin O, Demirors $\mathrm{H}$, et al. Analysis of modified oblique Keller procedure for severe hallux rigidus. Foot Ankle Int 2008;29(12):1203-1208. DOI: 10.3113/FAI.2008.1203. 
28. Patel HA, Kalra R, Johnson JL, et al. Is interposition arthroplasty a viable option for treatment of moderate to severe hallux rigidus? a systematic review and meta-analysis. Foot Ankle Surg 2019;25(5): 571-579. DOI: 10.1016/j.fas.2018.07.006.

29. Coughlin MJ, Shurnas PJ. Soft-tissue arthroplasty for hallux rigidus. Foot Ankle Int 2003;24(9):661-672. DOI: 10.1177/107110070302400902.

30. Cracchiolo All, Weltmer Jr JB, Lian G, et al. Arthroplasty of the first metatarsophalangeal joint with a double-stem silicone implant. Results in patients who have degenerative joint disease failure of previous operations, or rheumatoid arthritis. J Bone Joint Surg Am 1992;74-A(4):552-563. DOI: 10.2106/00004623-199274040-00011.

31. Pulavarti RS, McVie JL, Tulloch CJ. First metatarsophalangeal joint replacement using the bio-action great toe implant: intermediate results. Foot Ankle Int 2005;26(12):1033-1037. DOI: 10.1177/107110070502601206.

32. Gibson JN, Thomson CE. Arthrodesis or total replacement arthroplasty for hallux rigidus: a randomized controlled trial. Foot Ankle Int 2005;26(9):680-690. DOI: 10.1177/107110070502600904.

33. Dülgeroğlu TC, Metineren H. Treatment of end-stage hallux rigidus using total joint arthroplasty: a short-term clinical study. J Foot Ankle Surg 2017;56(5):1047-1051. DOI: 10.1053/j.jfas.2017.05.017.

34. Park $\mathrm{YH}$, Jung JH, Kang SH, et al. Implant arthroplasty versus arthrodesis for the treatment of advanced hallux rigidus: a metaanalysis of comparative studies. J Foot Ankle Surg 2019;58(1):137-143. DOI: 10.1053/j.jfas.2018.08.045.
35. Stibolt RD, Patel HA, Lehtonen EJ, et al. Hemiarthroplasty versus total joint arthroplasty for hallux rigidus: a systematic review and meta-analysis. Foot Ankle Spec 2019;12(2):181-193. DOI: 10.1177/1938640018791017.

36. Glazebrook M, Blundell CM, O'Dowd D, et al. Midterm outcomes of a synthetic cartilage implant for the first metatarsophalangeal joint in advanced hallux rigidus. Foot Ankle Int 2019;40(4):374-383. DOI: 10.1177/1071100718815469.

37. Cassinelli SJ, Chen S, Charlton TP, et al. Early outcomes and complications of synthetic cartilage implant for treatment of hallux rigidus in the United States. Foot Ankle Int 2019;40(10):1140-1148. DOI: 10.1177/1071100719855049.

38. McNeil DS, Baumhauer JF, Glazebrook MA. Evidence-based analysis of the efficacy for operative treatment of hallux rigidus. Foot Ankle Int 2013;34(1):15-32. DOI: 10.1177/1071100712460220.

39. Kelikian AS. Technical considerations in hallux metatarsal-phalangeal arthrodesis. Foot Ankle Clin 2005;10(1):167-190. DOI: 10.1016/j. fcl.2004.11.002.

40. Politi J, John H, Njus G, et al. First metatarsal-phalangeal joint arthrodesis: a Biomechanical Assessment of stability. Foot Ankle Int 2003;24(4):332-337. DOI: 10.1177/107110070302400405.

41. Goucher NR, Coughlin MJ. Hallux metatarsophalangeal joint arthrodesis using dome-shaped reamers and dorsal plate fixation: a prospective study. Foot Ankle Int 2006;27(11):869-876. DOI: $10.1177 / 107110070602701101$. 